\title{
TeDia - A Telemedicine-Based Treatment Model for Inpatient and Interprofessional Diabetes Care
}

This article was published in the following Dove Press journal:

Diabetes, Metabolic Syndrome and Obesity: Targets and Therapy

\author{
Martin Röhling $\mathbb{D}^{1, *}$ \\ Marcus Redaélli ${ }^{2, *}$ \\ Dusan Simic ${ }^{2}$ \\ Kristina Lorrek ${ }^{2}$ \\ Christina Samel ${ }^{3}$ \\ Paul Schneider ${ }^{4}$ \\ Kerstin Kempf (D) \\ Stephanie Stock ${ }^{2}$ \\ Stephan Martin ${ }^{1,5}$
}

'West-German Center of Diabetes and Health, Düsseldorf Catholic Hospital

Group, Düsseldorf, Germany; ${ }^{2}$ Institute of Health Economics and Clinical Epidemiology, University Hospital of Cologne, Cologne, Germany; ${ }^{3}$ Institute of Medical Statistics and Computational Biology, Faculty of Medicine, University of Cologne, Cologne, Germany;

${ }^{4}$ Düsseldorf Catholic Hospital Group, Düsseldorf, Germany; ${ }^{5}$ Faculty of Medicine, Heinrich Heine University Düsseldorf, Düsseldorf, Germany

*These authors contributed equally to this work
Correspondence: Martin Röhling West-German Centre of Diabetes and Health, Düsseldorf Catholic Hospital Group, Hohensandweg 37, Düsseldorf 4059I, Germany

Tel +49 2II-56 6036076

Fax +49 2II-56 6036072

Email martin.roehling@vkkd-kliniken.de
Introduction: The proportion of hospitalized patients with diabetes as a secondary diagnosis increases continuously. Therefore, we have developed a team-based interprofessional and telemedicine-based diabetes management system named TeDia ("Telemedical Diabetology") and implemented it in an inpatient setting. The aim of the retrospective realworld study was to show the clinical impact of TeDia following its implementation.

Material and methods: TeDia is characterized by an interpersonal and telemedicine-based exchange of hospital routine data between specially trained nurses (“diabetes managers") and external diabetologists. It was implemented in three acute hospitals of the Düsseldorf Catholic Hospital Group in Düsseldorf, Germany. Clinical awareness of diabetes, diabetesrelated complications and diagnosis-related group (DRG)-based revenues were analyzed using ICD routine coding. Furthermore, the frequency of HbAlc determinations as well as hospitalization days were investigated.

Results: Before (2010), during (2012) and after the implementation of TeDia (2014), the number of patients with ICD coding for diabetes, decompensated diabetes, diabetic neuropathy, diabetic nephropathy as well as complicated diabetes increased by $+18 \%,+93 \%$, $+101 \%,+113 \%$ and $+89 \%$, respectively. Using the same DRG grouper, revenues increased by $+53 \%$ (from 27 (2013) to 42 (2014) DRG points). Frequency of HbA1c determinations rose by $+85 \%$, whereas the time for an average length of stay decreased by $-12 \%(-0,91$ days) in comparison to patients without diabetes.

Conclusion: TeDia improved clinical awareness for diabetes and its complications. This new treatment model increased revenues and reduced hospital days indicating enhanced treatment quality. Our findings emphasize the necessity of novel technologies in inpatient settings for the improvement of efficacy, safety and efficiency of diabetes care.

Keywords: telemedicine, diabetes, inpatient care

\section{Introduction}

Since its introduction in 2003 and 2004, the German diagnosis-related group (G-DRG) has been used to bill inpatient service. ${ }^{1}$ The DRG distinguishes between main (reason for inpatient stay) and secondary diagnoses. While patients with the main diagnosis diabetes receive quality-assured care according to their metabolic state, those with the secondary diagnosis diabetes are treated on the reason for the admission. Moreover, the prevalence of patients with a secondary diagnosis of diabetes has steadily increased in German hospitals in the past decades. ${ }^{2-4}$ Nevertheless, diabetes has lost its importance as a primary diagnosis for hospital admissions and changed into a predominant field of outpatient care at the same time. ${ }^{5}$ These changes have contributed to serious consequences, as recent studies 
show that uncontrolled hyperglycemia in hospitalized patients with or without a previous diagnosis of diabetes is associated with adverse outcomes and extended hospital days. ${ }^{6}$ Furthermore, it is known that adequate diabetes treatment can delay or even prevent complications resulting in a decrease of morbidity and mortality in addition to substantial cost savings. ${ }^{7-10}$ However, many diabetes patients do not receive adequate treatment, such as a treatment based on the "insulin sliding scale", 5 or no treatment at all. ${ }^{11}$ Therefore, a substantial proportion of patients remain undiagnosed during their hospital stay. ${ }^{12,13}$ Further factors, which are disadvantageous for an adequate inpatient diabetes treatment, comprise: (i) lack of time of physicians for providing diabetes-related information, ${ }^{14}$ (ii) an inefficient screening procedure, and (iii) coding of diabetes and its complications. ${ }^{15}$ However, there is evidence that the collaboration of an interprofessional and interdisciplinary team, consisting of physicians and non-physician staff, can improve patient education and diabetes care in an inpatient setting. ${ }^{16,17}$ Moreover, a recently published study demonstrated that a multidisciplinary team approach could reduce the rate of readmission into hospitals caused by acute glycaemic events. ${ }^{18}$ Additionally, previous studies have shown that appropriately trained or specialised non-medical staff can also provide high quality care in comparison to physicians. ${ }^{19-23}$ Based on this background, we have developed an interprofessional and telemedicine-based diabetes management system named TeDia ("telemedical diabetology") and implemented it in three hospitals of the Düsseldorf Catholic Hospital Group in Düsseldorf, Germany. In the present study we hypothesized that the implementation of TeDia: (i) improves awareness of diabetes, (ii) reduces hospital days as a consequence of improved treatment quality, and (iii) leads to improved revenues due to a better ICD coding.

\section{Materials and Methods TeDia System}

The TeDia system consists of two components: (1) screening for diabetes-related complications such as nephropathy or foot ulcers in known diabetes (secondary diagnosis) or screening for unknown diabetes through conspicuous laboratory findings or anamnesis, and (2) telemedical therapy support for diabetes and its complications during hospitalization. The inpatient care was provided by 2 senior physicians with additional diabetes qualifications (diabetologists $\hat{=}$ internists who are specially trained in diabetes) and 5 nurses who have been trained in diabetes (not academic). Approximately 50-60 patients were treated per day in the three acute hospitals, of which only $8-10 \%$ were not followed-up. The main reasons for not performing follow-ups were that family doctors did not want it or the patients had no interest in it. All patients with the secondary diagnosis diabetes were visited by nurses specially trained in diabetes ("diabetes managers") at their day of hospital admission. In addition to this, all patients were screened for undiagnosed diabetes by performing glucose monitoring at admission day. The diabetes managers conducted a personal interview with the patients with the secondary diagnosis diabetes including questions about diabetes history, current therapy and diabetes-related complications. These data were transferred in a structured manner into the database ('DiabetesInformation-System (DIS)) of TeDia. Moreover, foot inspection and screening for diabetic neuropathy were performed and the results were incorporated into an electronic file in DIS. In case of insulin therapy, injections sites were inspected. Furthermore, diabetes managers had the permission to request $\mathrm{HbA} 1 \mathrm{c}$, blood lipid and microalbuminuria measurements. All diabetes-related laboratory values were automatically transferred to TeDia, including the blood glucose values at the wards. These data were checked by a team of diabetologist and specialized nurses of the West-German Centre of Diabetes and Health in Düsseldorf, Germany, which had an external access to DIS. After checking the data, diabetes therapy was adjusted by the diabetologists, when necessary. In case of a conventional insulin therapy or decompensated metabolic control, patients received a basal-bolus regimen. Insulin adaptation plans were created and adapted day-today telemedically based on the current blood glucose monitoring at the ward during the hospital stay by diabetologists. The diabetes managers at the ward ensured that adaptations of insulin dosing were implemented and discussed with the resident physician. All internal and external information, including changes of diabetes therapy, were documented and transferred to TeDia. A comprehensive report for the family doctors was prepared at the end of the hospital stay. The TeDia system and its interrelationships are illustrated in Figure 1.

\section{Study Design and Population}

The design of a retrospective observational study in a prepost-interventional format was chosen to investigate the 
- Therapy adjustment based on current laboratory and clinical data

- Interdisciplinary exchange

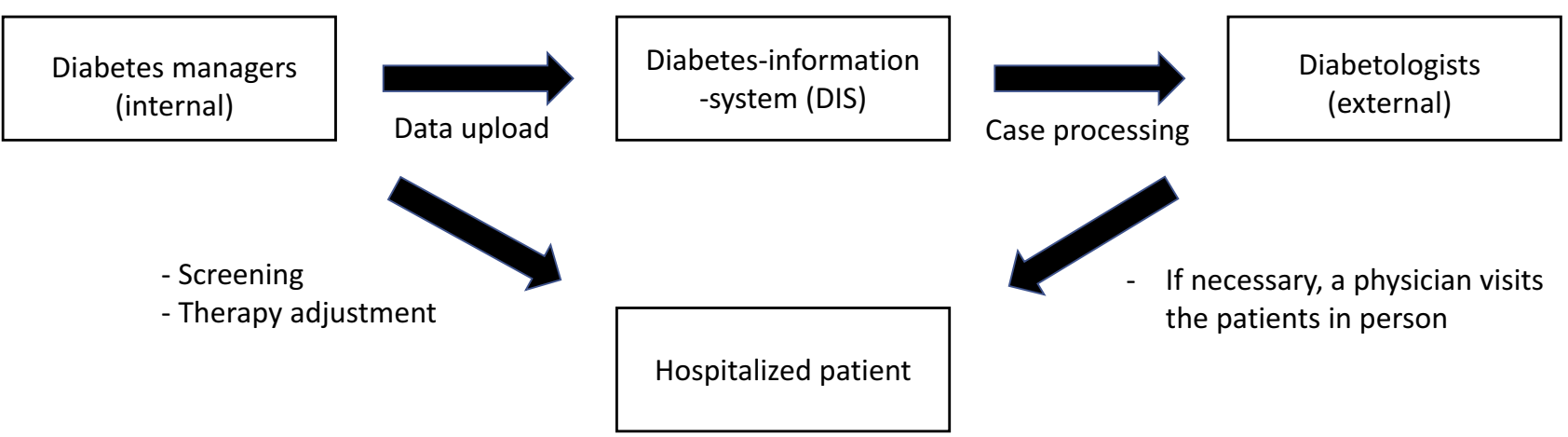

Figure I Model of the TeDia system and its interrelationships. Diabetes managers are nurses specially trained in diabetes.

effects of telemedical diabetes care. The TeDia system was implemented in three acute hospitals of the Düsseldorf Catholic Hospital Group from 2011 to 2012. General routine data were analyzed to evaluate TeDia using age, sex, hospital division, diagnosis-related group (DRG) coding, time of hospital stay and laboratory data (e.g. HbA1c). These data were recorded from all patients with a secondary diagnosis of diabetes and an inpatient stay before (2010), during (2012), and after (2014) implementation of TeDia. We chose 2010 as baseline, as this year was a representative year for the three acute hospitals before the implementation of TeDia. The year 2012 can be seen as a midpoint of our study and further interpreted as a "learning year" for implementing all "TeDia processes" into daily clinical practice. Persons were verified as diabetes patients by either their medical or personal anamnesis as well as via HbAlc determination. HbAlc analyses were performed when one single elevated blood glucose value was larger than $150 \mathrm{mg} / \mathrm{dl}$ during the hospital stay. Calculations of G-DRG-based revenues were performed with the identical DRG grouper within the observation period. DRG coding guarantees - in principle fixed - prices for invoices and each DRG code has a specific economic case value, which is multiplied with a base rate which is up to now specific for each hospital. Furthermore, the study investigators had full access to all routine data. The present retrospective observational study was conducted in accordance with the current ethical standards laid down in the 2013 Declaration of Helsinki. Approval of the research protocol was obtained from the ethics committee of the Medical Council North Rhine (Ärztekammer Nordrhein No. 2011294).

\section{Outcomes}

In order to analyze the efficiency and quality of care provided by TeDia, the change in service delivery in the secondary diagnosis of diabetes and the quality of coding for the diagnosis of diabetes were observed. In detail, we investigated our data regarding: (i) absolute and relative change of the secondary diagnosis diabetes, (ii) uncomplicated and complicated as well as (iii) decompensated diabetes. Furthermore, we have analyzed the coding of (iv) diabetic nephropathy and diabetic neuropathy diagnoses as well as the amount of (v) HbAlc determinations, which can be used as a surrogate marker for care quality. To evaluate the economic change due to the implementation of TeDia, we analyzed the (vi) absolute and relative change of time for a hospital stay as well as the (vii) revenues as a result of an improved coding.

All treatment cases per calendar year were taken into account. Patients who have been re-admitted within 30 days of discharge were treated as the same treatment case. Patients who were above the turn of the year were assigned to the payroll year and, as consequence, double treatment cases are excluded.

\section{Statistics}

Data are presented as means or percentages. We did not perform a priori sample size calculation, as we intended to examine all patients with a secondary diagnosis diabetes 
within the implementation phase of TeDia from 2010 to 2014. The collective was recruited from three acute hospitals. Unlike special clinics, these hospitals do not allow patients to plan ahead. Therefore, no standardization was possible. As a result, an exclusively descriptive evaluation without significance representation was chosen. Thus, only clinical trends were described in this article. All analyses were performed using SPSS 22.0 (SPSS Inc., Chicago, IL, USA) and GraphPad Prism 6.04 (GraphPad Software, San Diego, CA, USA).

\section{Results}

The baseline survey (before the introduction of TeDia) is 2010, from which, with the exception of the coding of "uncomplicated diabetes", a steady increase can be read over the dates (2012 and 2014) (Figure 2). The number of secondary diagnoses "diabetes" rose by $18 \%$ or by 1028 treatment cases in 2014 compared with the base year 2010. The diagnoses "decompensated diabetes" or "complicated diabetes" also almost doubled. The diagnosis "complicated diabetes" increased by $89 \%$ or 1468 treatment cases, "decompensated diabetes" by $93 \%$ or 960 treatment cases. On the other hand, the diagnosis "uncomplicated diabetes" decreased continuously by 605 treatment cases. This corresponds to a decrease of $13 \%$ from the base year 2010 to 2014. For a comparison of the coding change of the study collective, reference is made to the prevalence compared to the number of treatment cases. While treatment cases increased by 4\% between 2010 and 2014, diabetes was diagnosed $16 \%$ more frequently during the same period.

In addition, the TeDia system also increased the identification rate of diabetes-related complications such as neuropathy and nephropathy. The identification rate of neuropathy increased by $+101 \%$ or 273 cases from 2010 to 2014. Diabetes-related nephropathy was more than doubled with an increase of $+113 \%$ and 624 cases respectively. After adjustment of the number of diagnosed diabetes-related complications to the frequency of the secondary diagnosis diabetes, the frequency of the complications considered here per diabetes diagnosis increased by $+4 \%,+8 \%$ and $+12 \%$ respectively for neuropathy, nephropathy and decompensated diabetes (Supplementary Figure 1).

A look at the length of hospital stay shows that the trend towards shorter hospital stays among patients with diabetes is stronger. While a decrease of $9 \%$ or 0.54 days can be observed in patients without diabetes, patients with diabetes have a shortened length of stay of $12 \%$ or 0.91 days (Figure 3). Accordingly, the revenue for patients with diabetes also shifted. The cost weight points (G-DRG points) increased relatively (absolutely) by $+53 \%$ (from 27.36 (2013) to 41.95 (2014) DRG points) and the frequency of $\mathrm{HbA1c}$ determinations rose relatively (absolutely $)$ by $+85 \%(n=6900)$ as illustrated in Figure 4 .

\section{Discussion}

In the present study, the implementation of TeDia, a team-based interprofessional and telemedicine-based diabetes management system, led to an improved awareness of diabetes and its complications in an inpatient setting. Previously published works ${ }^{24}$ underpin our findings showing that a transition from a paper-based approach to a virtual glucose management service can reduce the proportion of hyperglycemic and hypoglycemic episodes in patients with diabetes by $-39 \%$ and $-36 \%$, respectively. ${ }^{25,26}$

Furthermore, the results indirectly indicate an improved inpatient diabetes treatment quality due to the reduction of the length of hospital stay in patients with diabetes compared to patients without diabetes. These findings are supported by another telemedicine-based intervention study, which took place in an outpatient setting. ${ }^{27}$ In this study, a telemedicine-based intervention with standard care led to a reduction in length of hospital stay compared to a control group with standard care in patients with chronic diseases (e.g. diabetes). ${ }^{27}$

Moreover, an important factor for the positive changes following the TeDia implementation could be the beneficial interaction between physicians and physician assistants, nurses or other health professionals. In line with this, studies have shown that a team-based and interprofessional diabetes care can effectively improve the treatment of patients with type $1^{28}$ and type 2 diabetes. $^{29}$

The West-German Centre of Diabetes and Health has developed a telemedicine-based system that considers all forms of diabetes and its complications. Given the complexity of diabetes, a variety of health care professionals, such as general practitioners or family doctors, diabetologists, nurses, dietitians, and podiatrists are needed to ensure an adequate and complete diabetes treatment. The present study indicates that appropriately trained medical staff can safely provide and improve inpatient diabetes care quality. An extended role of non-physician staff leads to more available time for patient care and allows 
A
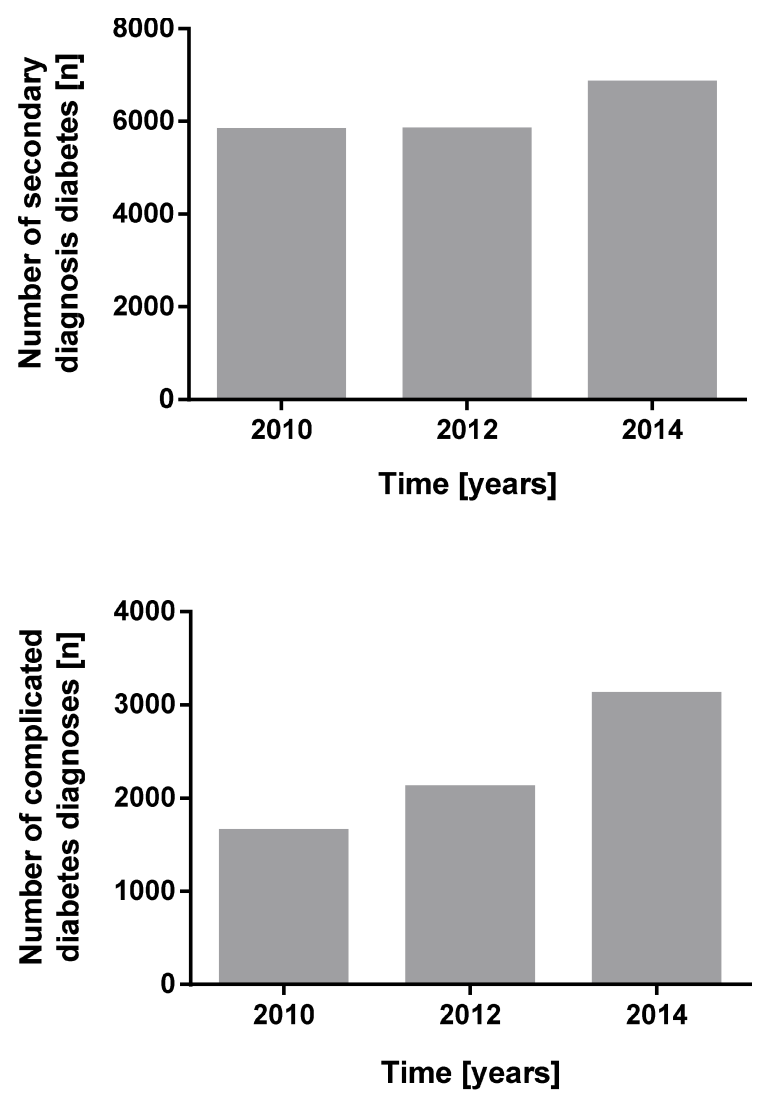

$\mathbf{E}$

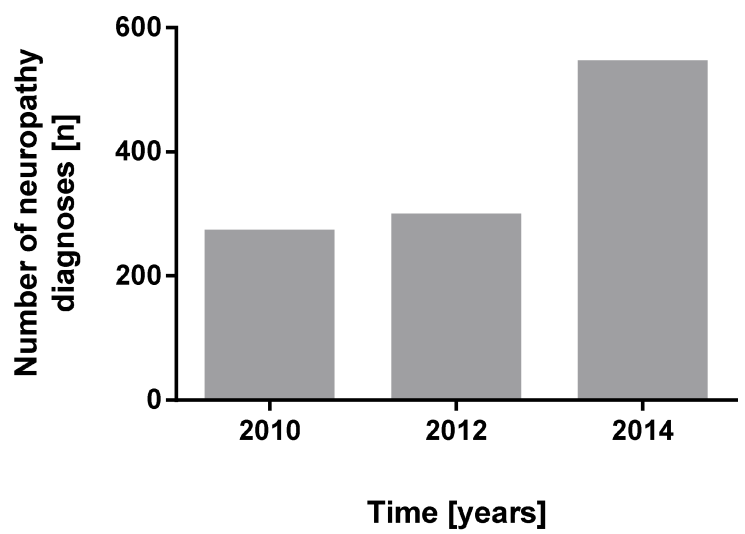

B
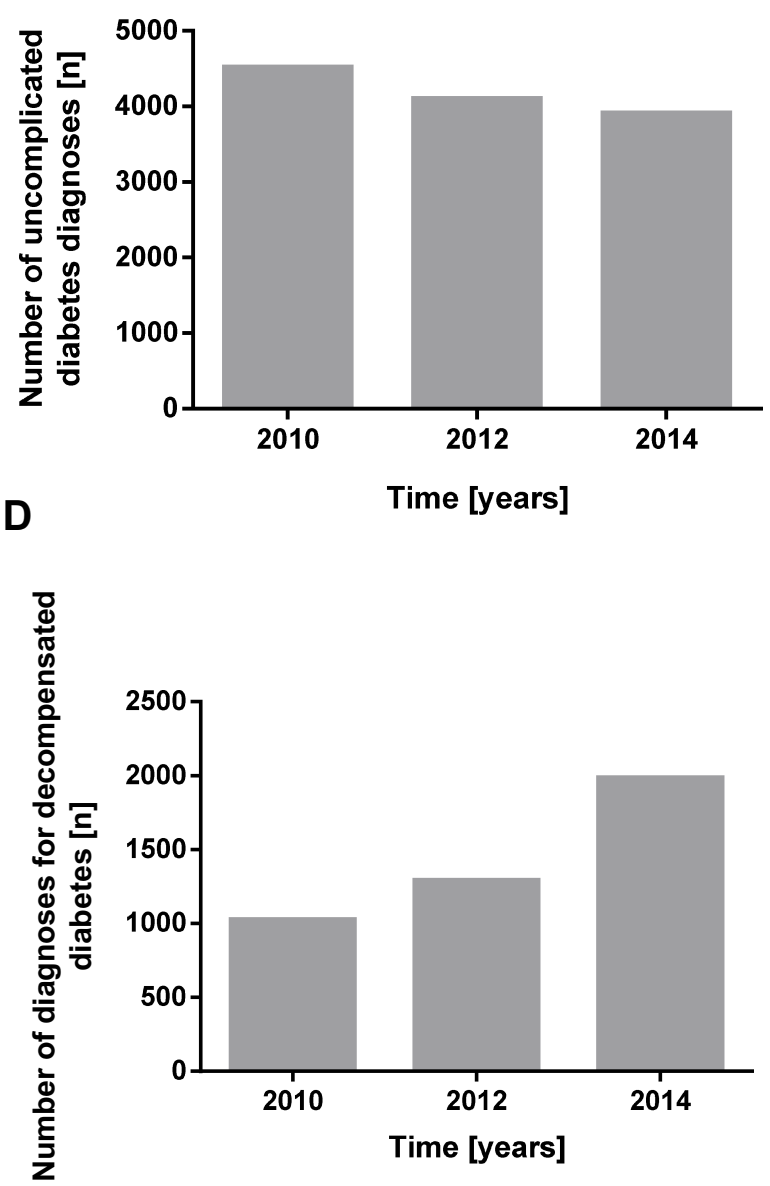

$\mathbf{F}$

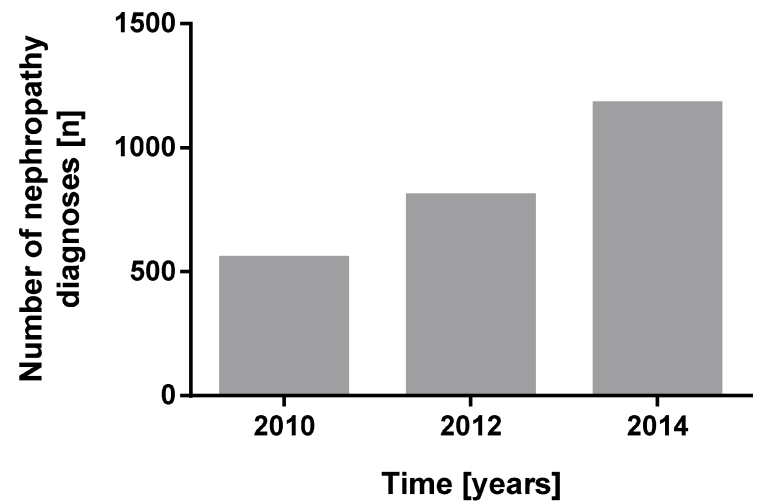

Figure 2 Frequency of diabetes-related diagnoses. Illustrated are absolute changes from 2010 to 2014 regarding (A) secondary diagnosis diabetes, (B) uncomplicated diabetes, $(\mathbf{C})$ complicated diabetes, (D) decompensated diabetes, (E) neuropathy and (F) nephropathy.

physicians to focus on more complex medical issues. ${ }^{30} \mathrm{At}$ the same time, non-physician team members can take over the tasks of providing diabetes education and screening of newly hospitalized patients. ${ }^{31}$
A further benefit of the implementation of TeDia was that it increased the revenues for the hospitals due to a better ICD coding and a reduction of length of hospital stay. To date, global diabetes-related costs are estimated to 
A

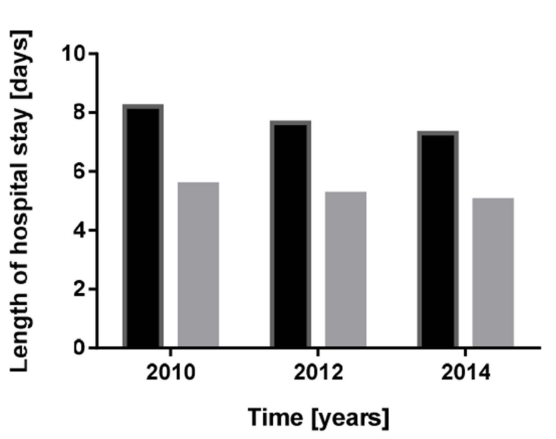

B

Patients with diabetes Patients without diabetes

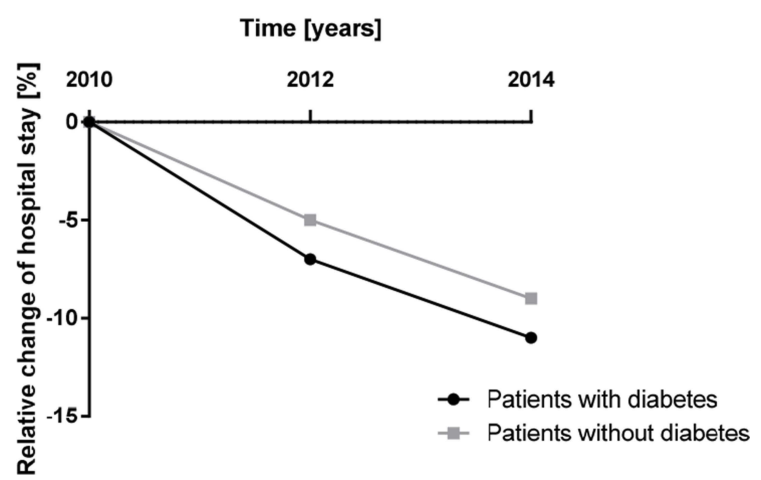

Figure 3 Length and relative change of hospital stay. Illustrated are the (A) absolute values and (B) the relative change of time from 2010 to 2014 regarding the average hospital stay for patients with or without diabetes.

A

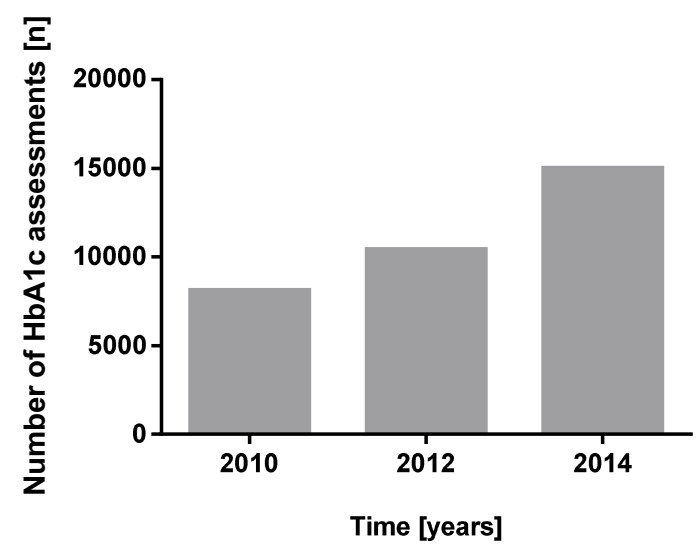

B

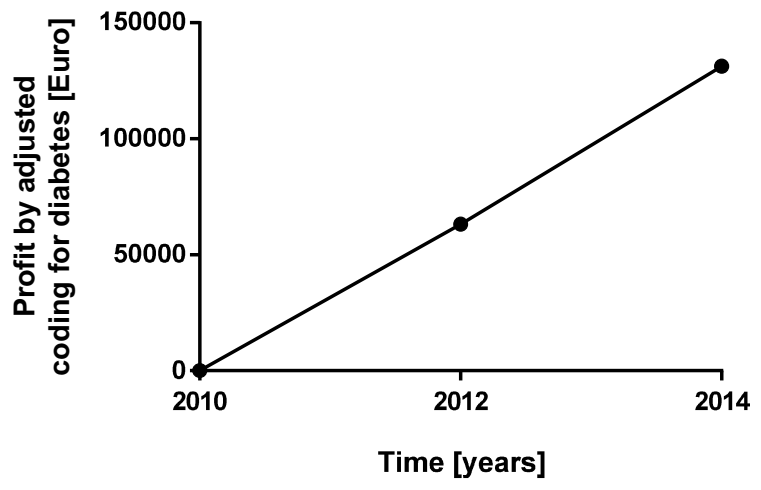

Figure 4 Treatment quality and revenues. Both graphs show the (A) absolute change of HbAlc determination and (B) revenues by adjusted coding from 2010 to 2014.

be around $12 \%$ of the entire health care expenditures per year ${ }^{32}$ with the largest proportions of costs related to the treatment of diabetes complications. ${ }^{33,34}$

It is known that adequate diabetes treatment can delay or even prevent diabetes-related complications resulting in substantial cost savings. ${ }^{35,36}$ In the present study, cost savings were accompanied by an enhanced treatment quality. The increased number of HbAlc determinations as well as the decrease in length of hospital stay in patients with diabetes, even in comparison to non-diabetic patients, suggest that TeDia might improve the inpatient treatment and process quality. Moreover, the implementation of the basal-bolus regimen in the daily routine led to a further progress in therapy quality. Several studies have shown that the change from the sliding scale insulin treatment to the basal-bolus regimen contributes to an increased treatment quality in patients with diabetes. ${ }^{35-37}$
There are strengths and limitations in our study that should be mentioned. The present study was designed with a pre-post cross-sectional approach basing only on secondary data due to legal restrictions. Thus, the process evaluation in the present study for treatment quality was limited to the data regarding the amount of HbAlc determinations and average length of hospital stay, which have shown to be two reliable parameters. Future studies could also include more subjective data, such as opinions and perceptions of patients as well as quality of life data to reflect the influence of telemedicine and interprofessional intervention.

A further weakness lies in the fact that only a historical control group from the time before the introduction of TeDia could be used (2010). However, our study approach bases on real-world data which we tried to examine with this proof-of-principle study. At least the comparison of the average length of hospital stay between patients with 
or without diabetes might suggest a potential benefit of TeDia. Furthermore, the overall findings of our study indicate to be of clinical significance; particularly in regard to reducing or even preventing complications in the further course. Therefore, performing statistical analyses seem to be irrelevant, especially as this is a cross-sectional analysis as well as a proof-of-principle study. On the other hand, another study could show that an innovative diabetesspecific structure and process management, comparable to TeDia, improves clinical awareness for diabetes and increases revenues. ${ }^{37}$ Therefore, we assume that the findings of our study could be reproduced in any other inpatient setting. A possible source of error in this kind of study could be a selection bias, however, as described in detail in the method section, every patient with a potential impaired glucose level was confirmed with an $\mathrm{HbAlc}$ determination.

A major strength of the study is that we investigated the impact of TeDia in three different hospitals with a large cohort of diabetes patients in an inpatient setting. In the present study the diabetes prevalence with $16 \%$ lies above the general diabetes prevalence rate. ${ }^{38}$ The TeDia system was able to improve inpatient diabetes care, despite a limited number of diabetologists and specialized nurses. While the beneficial effect of telemedicine for diabetes treatment in different settings is already known, ${ }^{39-41}$ the present study shows, that a telemedicine-based system combined with an interprofessional teamwork approach can be successfully implemented in an inpatient setting.

\section{Conclusion}

The results of the investigated TeDia system indicate a beneficial effect of an interprofessional and telemedicinebased approach. The implementation of TeDia led to an improved care quality, which was ensured by qualified physicians and specially trained nurses. Moreover, our findings emphasize the necessity of novel technologies in an inpatient setting by improving efficacy, safety and efficiency of diabetes care. Rapid analyzes of clinical and laboratory data accelerate clinical decision-making and therapy adjustments in the hospital setting and improve inpatient diabetes care.

\section{Abbreviations}

G-DRG, German diagnosis-related group; HbAlc, hemoglobin A1c; TeDia, Telemedical Diabetology.

\section{Ethics Statement}

Approval of the research protocol was obtained from the ethics committee of the Medical Council North Rhine (Ärztekammer Nordrhein No. 2011294). No human or animal data were collected, only routine data were used.

\section{Availability of Data and Materials}

The data used to support the findings of this study are available from the corresponding author upon request.

\section{Acknowledgments}

We thank Yasemin Kempf for proofreading the paper.

\section{Author Contributions}

All authors contributed to data analysis, drafting or revising the article, gave final approval of the version to be published, and agree to be accountable for all aspects of the work.

\section{Funding}

The study was financially supported by Sanofi-Aventis Deutschland $\mathrm{GmbH}$. The funder had no influence on study design, data collection, data analysis, manuscript preparation and/or publication decisions.

\section{Disclosure}

Dr Kerstin Kempf reports personal fees from Verlag Kirchheim $\mathrm{GmbH}$ \& Co. KG, outside the submitted work; Prof. Dr. Stephan Martin reports grants from Sanofi Aventis, during the conduct of the study. The authors report no other conflicts of interest in this work.

\section{References}

1. Hoffmann GE, Schenker M, Kammann M, Meyer-Luerssen D, Wilke MH. The significance of laboratory testing for the German diagnosis-related group system-the systematic evaluation of comorbidities of relevance to case reimbursement and continued development of the DRG Watchdog software. Clin Lab. 2004;50:599-607.

2. Umpierrez GE, Pasquel FJ. Management of inpatient hyperglycemia and diabetes in older adults. Diabetes Care. 2017;40:509-517. doi:10.2337/dc16-0989

3. Schneider AL, Kalyani RR, Golden S, et al. Diabetes and prediabetes and risk of hospitalization: the Atherosclerosis Risk in Communities (ARIC) study. Diabetes Care. 2016;39:772-779. doi:10.2337/dc151335

4. Muller N, Heller T, Freitag MH, et al. Healthcare utilization of people with type 2 diabetes in Germany: an analysis based on health insurance data. Diabet Med. 2015;32:951-957. doi:10.1111/dme.2015.32. issue-7

5. Abourizk NN, Vora CK, Verma PK. Inpatient diabetology. The new frontier. J Gen Intern Med. 2004;19:466-471. doi:10.1111/j.15251497.2004.30133.x 
6. Horton WB, Subauste JS. Top 10 facts to know about inpatient glycemic control. Am J Med. 2016;129:139-142. doi:10.1016/j. amjmed.2015.10.007

7. Buehler L, Fayfman M, Alexopoulos AS, et al. The impact of hyperglycemia and obesity on hospitalization costs and clinical outcome in general surgery patients. $J$ Diabetes Complications. 2015;29:1177-1182. doi:10.1016/j.jdiacomp.2015.07.027

8. Tonks KT, Jones GR, McGeechan K, Campbell LV. Hyperglycaemia in hospital inpatients: still a sticky situation. Intern Med J. 2010;40:521-526. doi:10.1111/j.1445-5994.2010.02197.x

9. Mendez CE, Mok KT, Ata A, Tanenberg RJ, Calles-Escandon J, Umpierrez GE. Increased glycemic variability is independently associated with length of stay and mortality in noncritically ill hospitalized patients. Diabetes Care. 2013;36:4091-4097. doi:10.23 $37 / \mathrm{dc} 12-2430$

10. Holman N, Hillson R, Young RJ. Excess mortality during hospital stays among patients with recorded diabetes compared with those without diabetes. Diabet Med. 2013;30:1393-1402. doi:10.1111/ dme.2013.30.issue-12

11. El Khoury G, Mansour H, Kabbara W, Chamoun N, Attalah N, Salameh P. Hyperglycemia in hospitalized diabetic non-critically ill patients: prevalence, correlates, management and nurses' attitudes. Curr Diabetes Rev. 2018.

12. Dall TM, Narayan KV, Gillespie KB, et al. Detecting type 2 diabetes and prediabetes among asymptomatic adults in the United States: modeling American Diabetes Association versus US Preventive Services Task Force diabetes screening guidelines. Popul Health Metr. 2014;12:1. doi:10.1186/1478-7954-12-12

13. Rathmann W, Haastert B, Icks AA, et al. High prevalence of undiagnosed diabetes mellitus in Southern Germany: target populations for efficient screening. The KORA survey 2000. Diabetologia. 2003;46:182-189. doi:10.1007/s00125-002-1025-0

14. Østbye T, Yarnall KS, Krause KM, Pollak KI, Gradison M, Michener JL. Is there time for management of patients with chronic diseases in primary care? Ann Fam Med. 2005;3:209-214. doi:10. 1370/afm. 310

15. Kufeldt J, Kovarova M, Adolph M, et al. Prevalence and distribution of diabetes mellitus in a maximum care hospital: urgent need for HbA1c-screening. Exp Clin Endocrinol Diabetes. 2018;126:123-129. doi:10.1055/s-0043-112653

16. Hutchison RW. Treating diabetes in underserved populations using an interprofessional care team. J Interprof Care. 2014;28:568-569. doi:10.3109/13561820.2014.917408

17. Arevian M. The significance of a collaborative practice model in delivering care to chronically ill patients: A case study of managing diabetes mellitus in a primary health care center. $J$ Interprof Care. 2005;19:444-451. doi:10.1080/13561820500215095

18. Simmons D, Hartnell S, Watts J, et al. Effectiveness of a multidisciplinary team approach to the prevention of readmission for acute glycaemic events. Diabet Med. 2015;32:1361-1367. doi:10.1111/dme.2015.32.issue-10

19. Machado M, Bajcar J, Guzzo GC, Einarson TR. Sensitivity of patient outcomes to pharmacist interventions. Part I: systematic review and meta-analysis in diabetes management. Ann Pharmacother. 2007;41:1569-1582.

20. Laurant M, Reeves D, Hermens R, Braspenning J, Grol R, Sibbald B. Substitution of doctors by nurses in primary care. Cochrane Database Syst Rev. 2005;CD001271.

21. Horrocks S, Anderson E, Salisbury C. Systematic review of whether nurse practitioners working in primary care can provide equivalent care to doctors. BMJ. 2002;324:819-823. doi:10.1136/bmj.324.7341.819

22. Wubben DP, Vivian EM. Effects of pharmacist outpatient interventions on adults with diabetes mellitus: a systematic review. Pharmacotherapy. 2008;28:421-436. doi:10.1592/phco.28.4.421

23. Loveman E, Royle P, Waugh N. Specialist nurses in diabetes mellitus. Cochrane Lib. 2003.
24. Mirhoseiny S, Geelvink T, Martin S, Vollmar HC, Stock S, Redaelli M. Does task delegation to non-physician health professionals improve quality of diabetes care? Results of a scoping review. PLoS ONE. 2019;14:e223159. doi:10.1371/journal.pone.0223159

25. Rushakoff RJ, Rushakoff JA, Kornberg Z, MacMaster HW, Shah AD. Remote monitoring and consultation of inpatient populations with diabetes. Curr Diab Rep. 2017;17:70. doi:10.1007/s11892-017-0896-x

26. Rushakoff RJ, Sullivan MM, MacMaster HW, et al. Association between a virtual glucose management service and glycemic control in hospitalized adult patients: an observational study. Ann Intern Med. 2017;166:621-627. doi:10.7326/M16-1413

27. Steventon A, Bardsley M, Billings J, et al. Effect of telehealth on use of secondary care and mortality: findings from the whole system demonstrator cluster randomised trial. BMJ. 2012;344:e3874. doi:10. 1136/bmj.e3874

28. Mendez CE, Umpierrez GE. Management of Type 1 diabetes in the hospital setting. Curr Diab Rep. 2017;17:98. doi:10.1007/s11892-0170919-7

29. Laurant $M$, van der Biezen $M$, Wijers $N$, Watananirun $K$, Kontopantelis E, van Vught AJ. Nurses as substitutes for doctors in primary care. Cochrane Database Syst Rev. 2018;7:Cd001271.

30. Yarnall KSH, Østbye T, Krause KM, Pollak KI, Gradison M, Michener JL. Family physicians as team leaders: "Time" to share the care. Prev Chronic Dis. 2009;6(2):A59.

31. Lenz ER, Mundinger MO, Hopkins SC, Lin SX, Smolowitz JL. Diabetes care processes and outcomes in patients treated by nurse practitioners or physicians. Diabetes Educ. 2002;28:590-598. doi:10. $1177 / 014572170202800413$

32. International Diabetes Federation. IDF diabetes Atlas Seventh Edition; 2015. Available from: http://www.diabetesatlas.org. Accessed June 15, 2016.

33. Tamayo T, Rosenbauer J, Wild S, et al. Diabetes in Europe: an update. Diabetes Res Clin Pract. 2014;103:206-217. doi:10.1016/j. diabres.2013.11.007

34. Li R, Bilik D, Brown MB, et al. Medical costs associated with type 2 diabetes complications and comorbidities. Am J Manag Care. 2013; 19:421.

35. Abdullah MM, Gyles CL, Marinangeli CP, Carlberg JG, Jones PJ. Cost-of-illness analysis reveals potential healthcare savings with reductions in type 2 diabetes and cardiovascular disease following recommended intakes of dietary fiber in Canada. Front Pharmacol. 2015;6:167. doi:10.3389/fphar.2015.00167

36. Li R, Zhang P, Barker LE, Chowdhury FM, Zhang X. Costeffectiveness of interventions to prevent and control diabetes mellitus: a systematic review. Diabetes Care. 2010;33:1872-1894. doi:10. 2337/dc10-0843

37. Tafel J, Buerstell M, Leippert C, Benack E, Stelling A, Jaeger C. Quality of patient care and an attempt to improve the care of diabetic inpatients in a german hospital. A trial to optimize inhouse processes, quality assurance and cost efficiency. Diabetologie. 2011;6:387-394.

38. Tamayo T, Schipf S, Meisinger C, Schunk M, Maier W, Herder C, Roden M, Nauck M, Peters A, Völzke H, Rathmann W. Regional differences of undiagnosed type 2 diabetes and prediabetes prevalence are not explained by known risk factors. PLoS One 2014; 9: e113154

39. Kempf K, Altpeter B, Berger J Reuß O, Fuchs M, Schneider M, Gärtner B, Niedermeier K, Martin S. Efficacy of the Telemedical Lifestyle intervention Program TeLiPro in Advanced Stages of Type 2 Diabetes: A Randomized Controlled Trial. Diabetes Care. 2017; 40: 863-871

40. Siminerio L, Ruppert K, Huber K, Toledo FG. Telemedicine for Reach, Education, Access, and Treatment (TREAT): linking telemedicine with diabetes self-management education to improve care in rural communities. Diabetes Educ. 2014; 40:797-805

41. Lepard MG, Joseph AL, Agne AA, Cherrington AL. Diabetes selfmanagement interventions for adults with type 2 diabetes living in rural areas: a systematic literature review. Curr Diab Rep. 2015; 15:608 


\section{Publish your work in this journal}

Diabetes, Metabolic Syndrome and Obesity: Targets and Therapy is an international, peer-reviewed open-access journal committed to the rapid publication of the latest laboratory and clinical findings in the fields of diabetes, metabolic syndrome and obesity research. Original research, review, case reports, hypothesis formation, expert opinion and commentaries are all considered for publication. The manuscript management system is completely online and includes a very quick and fair peer-review system, which is all easy to use. Visit $\mathrm{http}: / /$ www.dovepress.com/testimonials.php to read real quotes from published authors. 